Archive for

Organic Chemistry

Arkivoc 2018, part iv, 149-157

\title{
Microwave-assisted Michael-type addition of 1-(arylsulfonyl)-pyrroles and -indoles to methyl vinyl ketone using bismuth triflate as catalyst
}

\author{
Kelsey C. Miles, Bradley J. Kohane, Benjamin K. Southerland, and Daniel M. Ketcha*
}

Department of Chemistry, Wright State University, Dayton, OH 45435, USA

Email: Daniel.ketcha@wright.edu

Dedicated to Professor Gordon W. Gribble on the occasion of his retirement from Dartmouth College

Received 01-25-2019

Accepted 03-01-2018

Published on line 03-30-2018

\section{Abstract}

The Michael type addition of pyrroles and indoles bearing strongly electron-withdrawing N-protecting groups (e.g., arylsulfonyl-, benzoyl-) to methyl vinyl ketone have not been previously reported. We find that such alkylative processes can be effected in low to moderate yields using bismuth triflate as catalyst in conjunction with microwave irradiation.

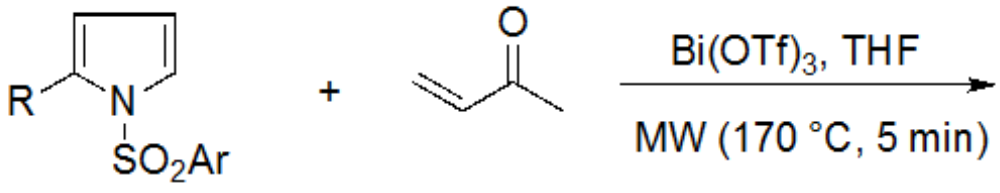<smiles>[R]c1ccc(CCC(C)=O)n1S(=O)(=O)O[Na]</smiles><smiles>[R]c1cc2ccccc2n1[S+](=O)O[Na]</smiles><smiles>C=CC(C)=O</smiles><smiles>[R]c1c(CCC(C)=O)c2ccccc2n1S(=O)(=O)O[Na]</smiles>

Keywords: Michael-type addition, microwave assisted, bismuth triflate, 1-(arylsulfonyl)pyrroles, 1-(phenylsulfonyl)indoles 


\section{Introduction}

In light of the diverse biological activities exhibited by molecules possessing an indole ${ }^{1-4}$ or pyrrole ${ }^{5}$ ring and their recognized status as 'privileged structures', methods for the synthesis and selective functionalization ${ }^{6,7}$ of these heterocycles continue to attract the attention of the synthetic community. Due to the relatively high reactivity of these heterocycles (especially of pyrrole) and the consequential lack of selectivity observed in many of their reactions, early protection of the ring nitrogen can play a pivotal role in synthetic planning, since the protecting group can serve to site-direct substitution as well as to attenuate the reactivity of this $\pi$ excessive ring system. Noteworthy in this regard is the $N$-phenylsulfonyl protecting group. Whereas addition of electrophiles to the C-2 $(\alpha)$ position of pyrrole is the most characteristic reaction of this heterocycle, $N$ (phenylsulfonyl)pyrroles display an ambivalent reactivity that allows for $\alpha$ - or $\beta$-substitution depending upon conditions. $^{8}$ However, this description is an oversimplification, for this selectivity is subject to a number of recognized and unrecognized parameters including: solvent, catalyst, temperature, stoichiometry, nature of electrophile, substituents on the pyrrole, and the addition of additives. One hitherto unexamined reaction with this substrate involves reaction with Michael acceptors.

The conjugate type addition of indoles and pyrroles to Michael acceptors represents a facile and direct route to the corresponding alkylated derivatives. Although first demonstrated upon indole employing protic acids, ${ }^{9}$ montmorillonite ${ }^{10}$ as well as doped clays $\left(\mathrm{AlCl}_{3}, \mathrm{FeCl}_{3} \text { and } \mathrm{ZnCl}_{2}\right)^{11}$ have been shown to be effective for reaction with methyl vinyl ketone (MVK) and methyl acrylate in dichloromethane (DCM). The first application of the rare earth class of catalysts to this end was conducted by Harrington and Kerr, who found that the treatment of indoles with a variety of electron-deficient olefins under the influence of $\mathrm{Yb}(\mathrm{OTf})_{3}-3 \mathrm{H}_{2} \mathrm{O}$ in acetonitrile leads to alkylation of the indoles at the 3-position. ${ }^{12}$ The authors noted that a number of Michael acceptors such as phenyl vinyl sulfone, ethyl cinnamate, methyl acrylate, acrylonitrile and several $\alpha, \beta-$ unsaturated ketones failed to react under these conditions. Yadav reported the efficient conjugate addition of indoles to $\alpha, B$-unsaturated compounds in the presence of indium chloride in DCM. ${ }^{13}$ However, again, some Michael acceptors like methyl acrylate and acrylonitrile failed to react under these conditions. Bismuth nitrate $^{14}$ and bismuth triflate ${ }^{15}$ have also been shown effective in this process, and a simple survey of solvents (dichloromethane, dichloroethane, water, THF and acetonitrile) indicated that while all were useful, acetonitrile was found to be superior. ${ }^{16}$ Scandium catalysis of this transformation has been reported involving $\mathrm{Sc}(\mathrm{OTf})_{3}{ }^{17}$ and Lewis acid-surfactant combined catalysts in water ${ }^{18}$ and supercritical carbon dioxide, ${ }^{19}$ and enantioselectively with the use of a chiral ligand. ${ }^{20}$ Other catalyst systems employed for this process include iodine, ${ }^{21,22}$ bromine, ${ }^{23} \mathrm{BF}_{3} \cdot \mathrm{OEt}_{2}{ }^{24}$ cerium derivatives ${ }^{25,26}$ and gallium halides. ${ }^{27,28}$ Additionally, Kobayashi has developed a neutral catalyst system of silica-supported sodium benzenesulfonate with a hydrophobic ionic liquid for the Michael reactions of indoles in water. $^{29}$

Less work has been described on the analogous reactions of pyrroles. Notably, pyrroles have been found to undergo efficient conjugate addition to electron-deficient olefins in the presence of a catalytic amount of $\mathrm{InCl}_{3}$ to afford the Michael adducts. ${ }^{30}$ With increased reaction times and molar ratio of reactants, 2,5dialkylated products were obtained, while some Michael acceptors such as methyl acrylate and acrylonitrile, failed to react with pyrroles under these conditions. Additionally, $\mathrm{Y}(\mathrm{OTf})_{3}{ }^{31}$ and $\mathrm{Bi}\left(\mathrm{NO}_{3}\right)_{3}{ }^{32}$ have also been found effective for this purpose, as was copper bromide, ${ }^{33}$ Amberlyst-15, ${ }^{34}$ as well as aluminum dodecyl sulfate trihydrate in water. ${ }^{35}$ Bismuth trichloride has been utilized for this purpose at room temperature ${ }^{36}$ or under microwave irradiation or while supported on silica gel under solventless conditions. ${ }^{37}$ Additionally, the conjugate addition of pyrrole to electron-deficient nitro-olefins has been demonstrated under catalyst-free 
conditions, $^{38}$ using a combination of microwave heating and water, ${ }^{39}$ in the presence of silica gel ${ }^{40,41}$ or sulfuric acid modified silica catalysts. ${ }^{42,43}$

\section{Results and Discussion}<smiles></smiles>

\section{Scheme 1}

Due to the attenuated reactivity of pyrroles bearing strongly electron-withdrawing groups on the nitrogen atom, our initial efforts to effect Michael-type additions using 1-(arylsulfonyl)-pyrroles or -indoles as nucleophiles under thermal conditions resulted in only trace amounts of products, regardless of catalyst. The remarkable ability of microwave irradiation to enhance reaction rates ${ }^{44,45}$ indicated that a reinvestigation of this process was warranted. To this end, a set of parallel experiments was conducted involving reactions of 1(phenylsulfonyl)pyrrole $\left(1.2 \mathrm{mmol}\right.$ ) with MVK (2 equiv) under microwave irradiation for $5 \mathrm{~min}$ at $120^{\circ} \mathrm{C}$ using Montmorillonite $\mathrm{K} 10$ (MK-10) or metal triflate catalysts $(10 \mathrm{~mol} \%)$ in THF $(2.5 \mathrm{~mL})$ and the products were analyzed by GC/MS, Table 1.

Table 1. Survey of catalysts for MW addition of 1-(phenylsulfonyl)pyrrole (1) to MVK

\begin{tabular}{cccc}
\hline Trial $^{a}$ & Catalyst & ${\text { Monosubstitution }(\%)^{b}}$ & ${\text { Disubstitution }(\%)^{b}}^{b}$ \\
\hline 1 & Mont K10 (150 wt \%) & 9.4 & $<2$ \\
2 & $\mathrm{Bi}(\mathrm{OTf})_{3}$ & 49.82 & 7.51 \\
3 & $\mathrm{Sc}(\mathrm{OTf})_{3}$ & 8.95 & $<2$ \\
4 & $\mathrm{Y}(\mathrm{OTf})_{3}$ & $>2$ & 0 \\
5 & $\mathrm{Yb}(\mathrm{OTf})_{3}$ & 0 & 0 \\
6 & $\mathrm{InCl}_{3}$ & 0 & 0 \\
7 & $\mathrm{In}(\mathrm{OTf})_{3}$ & 0 & 0 \\
8 & $\mathrm{Bi}\left(\mathrm{NO}_{3}\right)_{3}$ & 0 & 0 \\
9 & $\mathrm{Bi}\left(\mathrm{NO}_{3}\right)_{3}$ Solventless $_{10}$ & 31.34 & 0 \\
\hline
\end{tabular}

${ }^{a}$ Reaction conditions: 1-(phenylsulfonyl)pyrrole (1.2 mmol), MVK (2 equiv), THF $(2.5 \mathrm{~mL}), \mathrm{MW}$ heating, $120{ }^{\circ} \mathrm{C}$, 5 min. ${ }^{b}$ Determined by GC/MS 
As seen from Table 1, the use of MK-10 (150 weight\%) provided poor conversion yielding a mixture of mono- and dialkylated products. Scanning various metal triflates (10 mol\%), it was found that while scandium triflate performed analogously to $\mathrm{MK}-10$, bismuth triflate appeared most effective in providing moderate yields of the monoalkylated adduct along with some of a disubstituted product. Whereas sulfamic acid and bismuth nitrate proved ineffective for the purpose, it is interesting to note that bismuth nitrate on silica gel (1:1 wt\%) under solventless conditions provided a moderate amount of monosubstitution with no discernible disubstitution.

Having established the comparative effectiveness of bismuth triflate in the case of 1-(phenylsulfonyl)pyrrole (1) as a representative substrate, varying the amount of catalyst from $5-20$ mol\% in $5 \%$ increments provided little variance in the yields of mono- and di-substituted products (GC/MS). Since it appeared that no advantage was to be gained at higher concentrations of catalyst, 10 mol\% was established as the standard condition for all subsequent trials. Increasing the reaction time of this prototype reaction from $300 \mathrm{sec}$ to $900 \mathrm{sec}$ led to an increase in the amount of disubstituted product along with some apparent trisubstitution adduct. Additional attempts at optimization involved examining the use of acetonitrile and nitromethane as alternative solvents under the same conditions of time and temperature. However these solvents provided the monosubstituted adduct in lower yields (e.g., $27.5 \%$ and $20.1 \%$ yield, respectively) along with ca. $2 \%$ of the di-adducts.

With the tentatively optimized reaction conditions for the Michael addition of 1-(phenylsulfonyl)pyrrole to MVK (i.e., 2 equiv MVK, $10 \mathrm{~mol} \% \mathrm{Bi}(\mathrm{OTf})_{3}$, THF, $120{ }^{\circ} \mathrm{C}, 5 \mathrm{~min}$ ), we explored the scope of this reaction with additional pyrroles and indoles bearing strongly electron withdrawing groups (Table 2 ).

Table 2. Bismuth triflate catalyzed Michael-type addition of N-substituted pyrroles and indoles (1a-1g)

Entry $\begin{gathered}\text { Pyrrole or Indole } \\ \begin{array}{c}\text { Temperature } \\ (\underline{0} \mathrm{C})\end{array}\end{gathered}$




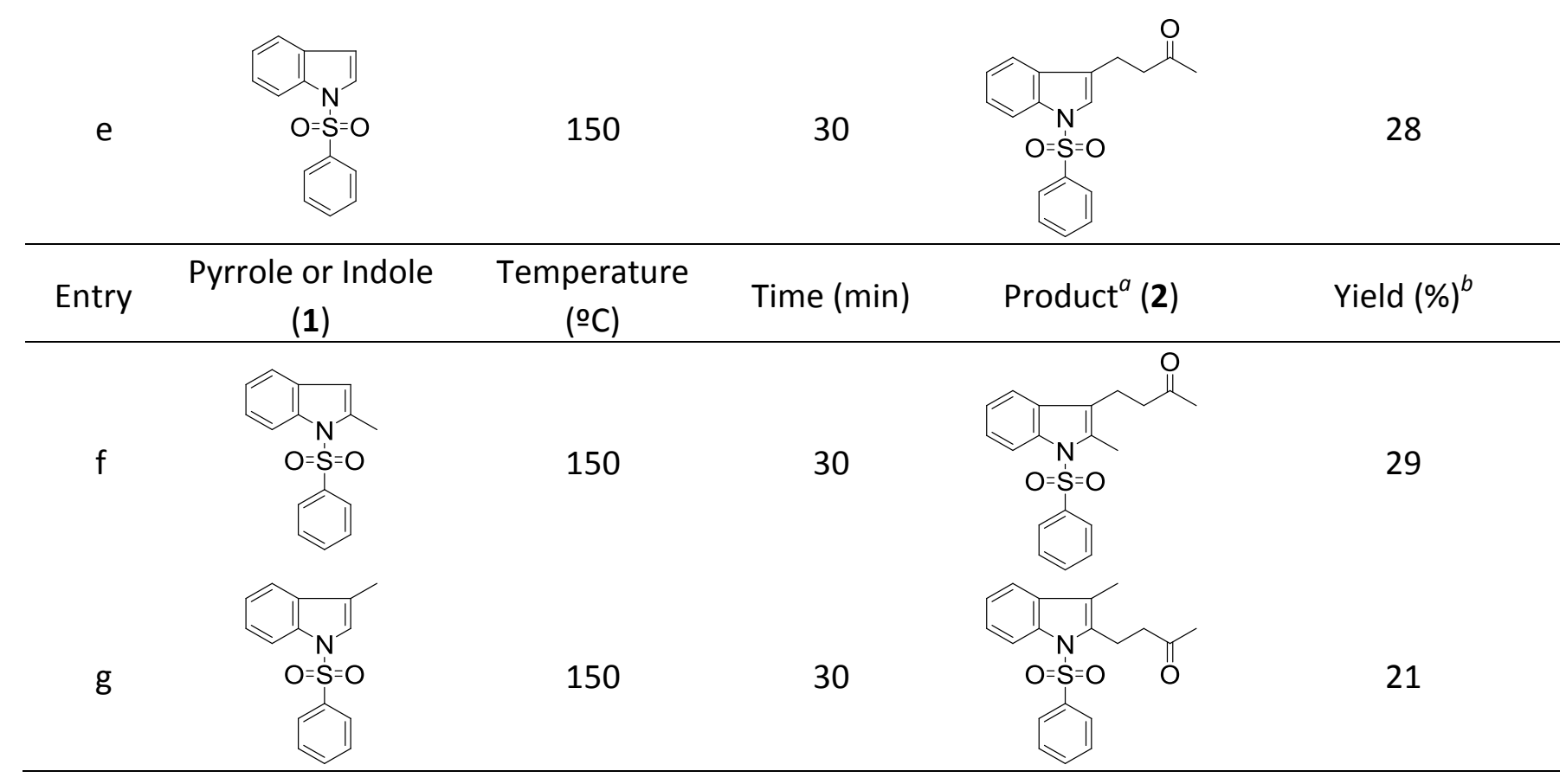

${ }^{a}$ Analyzed by GC/MS and NMR and either HRMS or $\mathrm{C}, \mathrm{H}$, and N combustion analysis. ${ }^{b}$ Isolated yields

In order to ascertain the generality of this reaction, an $N$-benzoyl protected pyrrole (1a) as well as two $N$ arylsulfonylpyrroles (16, 1c) were examined. ${ }^{46}$ A representative C-2 alkyl substituted-1-(phenylsulfonyl)pyrrole (e.g., benzyl, 1d) derivative prepared by an acylation/reductive deoxygenation protocol ${ }^{47}$ was also examined so as to establish that substitution occurred at the alternate $\alpha$-position. Finally, 1-(phenylsulfonyl)indole (1e) as well as its C-2 (1f) and C-3 methyl (1g) analogs ${ }^{48}$ were examined so as to establish that although C-3 alkylation is expected, a substituent at this site diverts the Michael-type addition to the normally less reactive C-2 site.

Although the survey of catalysts, solvents, time and temperature using $\mathbf{1 b}$ as a prototype were conducted at $120{ }^{\circ} \mathrm{C}$, optimal results for most of the substrates in Table 2 were achieved at $170{ }^{\circ} \mathrm{C}$, so this temperature was then employed for all examples shown in this Table. As can be seen, the Michael addition of these substrates to MVK could be achieved in all situations albeit in low to moderate yield. In the case of $\mathrm{N}$ protected pyrroles (1a-1d), monosubstituted adducts were isolated after column chromatography (no attempt was made to isolate other byproducts). Expectedly, the alkylation of 1-(phenylsulfonyl)indoles occurred at the C-3 position if unsubstituted (e.g., 1e-f) but at the C-2 position in the case of a 3-alkyl derivative (1g).

\section{Conclusions}

In efforts to expand the role of $\mathrm{N}$-arylsulfonyl protecting groups for the selective functionalization of indoles and pyrroles, the hitherto unreported Michael addition of these substrates bearing strongly electronwithdrawing substituents to MVK to is reported. Albeit the reactions were effected in low to moderate yields, the challenge of this type of process can be gleaned from one recently reported observation ${ }^{23}$ that 1 (phenylsulfonyl)indole was inert to such an alkylation when bromine was employed as catalyst. Our contribution thus expands the scope and limitations of reactions performed upon pyrroles and their benzo derivatives. $^{49}$ 


\section{Experimental Section}

General. Melting points were determined via the use of open capillaries with an Electrothermal melting point apparatus. Elemental analyses was performed by Midwest Microlab, Indianapolis, IN and High Resolution Mass Spectrometry was performed using a Thermo Scientific LTQ-FT instrument by University of Cincinnati Mass Spectroscopy Facility, Cincinnati, $\mathrm{OH}$. Elemental analysis results are within $+0.4 \%$ of the theoretical

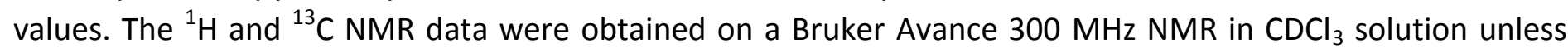
otherwise indicated. Chemical shifts for proton NMR are reported in $\delta$ (ppm) downfield from tetramethylsilane as an internal standard and ${ }^{13} \mathrm{C}$ NMR shifts were calibrated on the $\mathrm{CDCl}_{3}$ resonance at $77.23 \mathrm{ppm}$ or on the DMSO- $d_{6}$ resonance of 39.50 ppm. GC/MS measurements were performed using a Hewlett-Packard 6890 Series GC with auto injection and mass fragments are reported as mass per charge, $\mathrm{m} / \mathrm{z}$. The GC was coupled with a mass spectrometer with Hewlett-Packard 5973 mass selective detector/quadrupole system. Flash column (Silica Gel, Premium Rf, 200-400 mesh, Sorbent Technologies) and thin layer chromatography (TLC) were performed on silica gel with indicated solvent systems. All microwave reactions were performed in a CEM Discover 300 Watt system at the specified temperatures and times.

General procedure, exemplified with the synthesis of 4-[1-(phenylsulfonyl)-1H-pyrrol-2-yl]butan-2-one (2b) To a $10 \mathrm{~mL}$ microwave vial charged with THF $(2.5 \mathrm{~mL}$ ) was added pyrrole or indole (1.2 mmol), MVK (2 equiv) and bismuth triflate $(10 \mathrm{~mol} \%)$. The reaction vessel was sealed and heated under microwave irradiation in a CEM Discover 300 Watt microwave reactor for $5 \mathrm{~min}$ at $170{ }^{\circ} \mathrm{C}$ with a pre-stirring of $15 \mathrm{sec}$. The crude mixture was purified by column chromatography on silica gel (hexane:DCM 70:30) to afford pure 4-[(1phenylsulfonyl)pyrrol-2-yl]butan-2-one (2b). Mp 85-87 ${ }^{\circ} \mathrm{C} ; \mathrm{GC} / \mathrm{MS} \mathrm{m} / z 277\left(\mathrm{M}^{+}\right), 234(100 \%), 141,77 ;{ }^{1} \mathrm{H}$ NMR $\delta$ : $7.76(\mathrm{dt}, J 7.4 \mathrm{~Hz}, 1.5 \mathrm{~Hz}, 2 \mathrm{H}), 7.60(\mathrm{tt}, J 7.4 \mathrm{~Hz}, 1.5 \mathrm{~Hz}, 1 \mathrm{H}), 7.50(\mathrm{tt}, J 7.4, J 1.5 \mathrm{~Hz}, 2 \mathrm{H}), 7.29(\mathrm{q}, J 1.6 \mathrm{~Hz}$, $1 \mathrm{H}), 6.20(\mathrm{t}, J 3.3 \mathrm{~Hz}, 1 \mathrm{H}), 5.98(\mathrm{~m}, 1 \mathrm{H}), 2.93(\mathrm{t}, J 7.4,2 \mathrm{H}), 2.75(\mathrm{t}, J 7.4,2 \mathrm{H}), 2.11(\mathrm{~s}, 3 \mathrm{H}) ;{ }^{13} \mathrm{C} \mathrm{NMR}\left(\mathrm{CDCl} \mathrm{N}_{3}, 300\right.$ $\mathrm{MHz}) \delta:$ 207.4, 139.3, 134.3, 134.0, 129.6, 126.8, 122.9, 113.0, 111.8, 43.3, 30.0, 21.7. Anal. Calcd for $\mathrm{C}_{14} \mathrm{H}_{15} \mathrm{NO}_{3} \mathrm{~S}: \mathrm{C}, 60.63 ; \mathrm{H}, 5.45 ; \mathrm{N}, 5.05$. Found: $\mathrm{C}, 60.61 ; \mathrm{H}, 5.33 ; \mathrm{N}, 5.02 \%$.

4-(1-Benzoyl-1H-pyrrol-2-yl)butan-2-one (2a). GC/MS m/z $241\left(\mathrm{M}^{+}\right), 136,119,105$ (100\%), 77; ${ }^{1} \mathrm{H}$ NMR $\delta: 7.65$ (dt, J 7.3, 1.6 Hz, 2H), $7.53(\mathrm{tt}, J 7.3 \mathrm{~Hz}, 1.5 \mathrm{~Hz}, 1 \mathrm{H}), 7.42(\mathrm{tt}, J 7.3, J 1.5 \mathrm{~Hz}, 2 \mathrm{H}), 6.71(\mathrm{t}, J 2.5 \mathrm{~Hz}, 1 \mathrm{H}), 6.04$ $(\mathrm{m}, 2 \mathrm{H}), 3.17(\mathrm{t}, J 7.4 \mathrm{~Hz}, 2 \mathrm{H}), 2.78(\mathrm{t}, J \mathrm{~J} .4,2 \mathrm{H}), 2.10(\mathrm{~s}, 3 \mathrm{H}) ;{ }^{13} \mathrm{C} N M R$ o: 208.2, 169.6, 136.1, 134.5, 132.5, $129.9,128.6,123.7,113.0,110.8,43.7,30.0,23.2$. HRMS: $m / z$ calcd for $[M+H]^{+} \mathrm{C}_{15} \mathrm{H}_{16} \mathrm{NO}_{2}$ 242.11756, found 242.11761.

4-[1-(4-Nitrophenylsulfonyl)-1H-pyrrol-2-yl]butan-2-one (2c). Mp 117-119 ${ }^{\circ} \mathrm{C} ; \mathrm{GC} / \mathrm{MS}: \mathrm{m} / \mathrm{z} 322\left(\mathrm{M}^{+}\right), 279$, 186, $136(100 \%), 94,{ }^{1} \mathrm{H}$ NMR (DMSO- $d_{6}$ ) $\delta: 8.43$ (dt J 8.8, $2.0 \mathrm{~Hz}, 2 \mathrm{H}$ ) , 8.10 (dt J 8.8, $2.0 \mathrm{~Hz}, 2 \mathrm{H}$ ), $7.40(\mathrm{~m}, 1 \mathrm{H}$ ), $6.33(\mathrm{t}, J 3.3 \mathrm{~Hz}, 1 \mathrm{H}), 6.12(\mathrm{~m}, 1 \mathrm{H}), 2.82(\mathrm{t}, J 6.5 \mathrm{~Hz}, 2 \mathrm{H}), 2.77(\mathrm{t}, J 6.5 \mathrm{~Hz}, 2 \mathrm{H}), 2.09(\mathrm{~s}, 3 \mathrm{H}),{ }^{13} \mathrm{C}$ NMR (DMSO-d $\left.d_{6}\right)$ $\delta: 206.8,150.6,143.1,134.4,128.2,125.2,122.7,113.0,112.8,41.3,29.6,20.9$. Anal. Calcd for $\mathrm{C}_{14} \mathrm{H}_{14} \mathrm{~N}_{2} \mathrm{O}_{5} \mathrm{~S}: \mathrm{C}$, 52.17; $H, 4.38 ; N, 8.69$. Found: $C, 52.05 ; H, 4.46 ; N, 8.53 \%$.

4-[5-Benzyl-1-(phenylsulfonyl)-1H-pyrrol-2-yl]butan-2-one (2d). GC/MS: $m / z 367\left(\mathrm{M}^{+}\right), 310,226$ (100\%), 168,

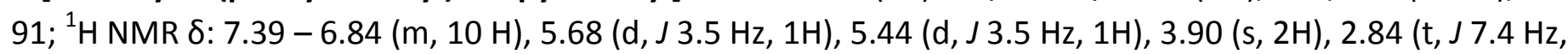

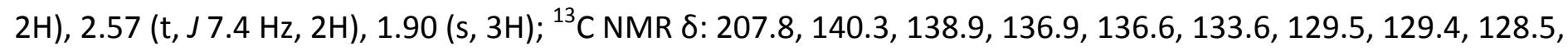
126.6, 126.2, 113.7, 112.0, 43.9, 35.4, 30.0, 23.5. HRMS: $\mathrm{m} / z$ calcd for $[\mathrm{M}+\mathrm{Na}]^{+} \mathrm{C}_{21} \mathrm{H}_{21} \mathrm{NO}_{3} \mathrm{SNa} 390.1141$, found 390.1140 . 
4-[1-(Phenylsulfonyl)-1H-indol-2-yl]butan-2-one (2e): GC/MS: $m / z \quad 327\left(\mathrm{M}^{+}\right), 284,270,144(100 \%), 77 ;{ }^{1} \mathrm{H}$ NMR $\delta: 7.98(d t, J 7.3 \mathrm{~Hz}, J 1.5 \mathrm{~Hz}, 1 \mathrm{H}), 7.84(\mathrm{dt}, J 7.4 \mathrm{~Hz}, J 1.5 \mathrm{~Hz}, 2 \mathrm{H}), 7.53-7.20(\mathrm{~m}, 7 \mathrm{H}), 2.93(\mathrm{t}, J 7.4 \mathrm{~Hz}$, $2 \mathrm{H}), 2.80(\mathrm{t}, J 7.4 \mathrm{~Hz}, 2 \mathrm{H}), 2.13(\mathrm{~s}, 3 \mathrm{H}) ;{ }^{13} \mathrm{C} N M R \delta: 207.6,138.3,135.4,133.8,130.8,129.3,126.8,125.0,123.4$, 122.9, 122.3, 119.5, 113.9, 42.8, 30.2, 18.9. Anal. Calcd for $\mathrm{C}_{18} \mathrm{H}_{17} \mathrm{NO}_{3} \mathrm{~S}: \mathrm{C}, 66.03 ; \mathrm{H}, 5.23 ; \mathrm{N}, 4.28$. Found: $\mathrm{C}$, $65.94 ; \mathrm{H}, 5.14 ; \mathrm{N}, 4.22 \%$.

4-[2-Methyl-1-(phenylsulfonyl)-1H-indol-3-yl]butan-2-one (2f). GC/MS: $\mathrm{m} / z 341\left(\mathrm{M}^{+}\right), 200(100 \%), 158,77 ;{ }^{1} \mathrm{H}$ NMR $\delta: 8.12(\mathrm{dt}, J 7.3 \mathrm{~Hz}, J 1.5 \mathrm{~Hz}, 1 \mathrm{H}), 7.66(\mathrm{dt}, J 7.4 \mathrm{~Hz}, J 1.5 \mathrm{~Hz}, 2 \mathrm{H}), 7.43(\mathrm{tt}, J 7.4 \mathrm{~Hz}, J 1.5 \mathrm{~Hz}, 1 \mathrm{H}), 7.36-$ $7.12(\mathrm{~m}, 5 \mathrm{H}), 2.79(\mathrm{t}, J 7.4 \mathrm{~Hz}, 2 \mathrm{H}), 2.57(\mathrm{t}, J 7.4 \mathrm{~Hz}, 2 \mathrm{H}), 2.46(\mathrm{~s}, 3 \mathrm{H}), 1.97(\mathrm{~s}, 3 \mathrm{H}) ;{ }^{13} \mathrm{C}$ NMR $\delta: 207.8,139.5$, $136.7,133.7,133.2,130.2,129.4,126.5,124.3,123.6,119.5,118.3,115.0,43.2,30.3,18.4,12.9$. HRMS: $\mathrm{m} / \mathrm{z}$ calcd for $[\mathrm{M}+\mathrm{Na}]^{+} \mathrm{C}_{19} \mathrm{H}_{19} \mathrm{NO}_{3} \mathrm{SNa} 364.0983$, found 364.0984 .

4-[3-Methyl-1-(phenylsulfonyl)-1H-indol-2-yl]butan-2-one (2g). GC/MS: m/z 341 (M+), 200 (100\%), 158, 143, 77; ${ }^{1} \mathrm{H}$ NMR $\delta: 8.31(\mathrm{~d}, J 7.3 \mathrm{~Hz}, 1 \mathrm{H}), 7.79(\mathrm{dt}, J 7.4 \mathrm{~Hz}, J 1.5 \mathrm{~Hz}, 2 \mathrm{H}), 7.62(\mathrm{tt}, J 7.4 \mathrm{~Hz}, J 1.5 \mathrm{~Hz}, 1 \mathrm{H}), 7.53-7.33$ $(\mathrm{m}, 5 \mathrm{H}), 3.33(\mathrm{t}, J 7.4 \mathrm{~Hz}, 2 \mathrm{H}), 3.04(\mathrm{t}, J 7.4 \mathrm{~Hz}, 2 \mathrm{H}), 2.29(\mathrm{~s}, 3 \mathrm{H}), 2.27(\mathrm{~s}, 3 \mathrm{H}) ;{ }^{13} \mathrm{C} N M R$ \&: 207.9, 138.9, 136.9, $135.7,133.8,131.7,129.4,126.4,124.7,123.9,118.9,118.0,115.3,44.1,29.9,20.9,9.1$. HRMS: $\mathrm{m} / z$ calcd for $[\mathrm{M}+\mathrm{Na}]^{+} \mathrm{C}_{19} \mathrm{H}_{19} \mathrm{NO}_{3} \mathrm{SNa} 364.0983$, found 364.0989 .

\section{Acknowledgements}

Financial support from the National Science Foundation - Course, Curriculum, and Laboratory Improvement (CCLI) Instructional Materials Development (DUE-0127240), Procter \& Gamble Fund, Curriculum Development Grant for Rapid Transfer of Knowledge and Technology is gratefully acknowledged.

\section{References}

1. Sundberg, R. J. "Indoles", Best Synthetic Methods Series, Academic Press, New York, 1996.

2. de Sa Alves, F. R.; Barreiro, E. J.; Fraga, C. A. M. Mini-Rev. Med. Chem. 2009, 9, 782-793. https://doi.org/10.2174/138955709788452649

3. Kaushik, N. K.; Kaushik, N.; Attri, P.; Kumar, N.; Kim, C. H.; Verma, A. K.; Choi, E. H. Molecules 2013, 18, 6620-6662.

https://doi.org/10.3390/molecules18066620

4. Bandini, M.; Eicholzer, A. Angew. Chem. Int. Ed. 2009, 48, 9608-9644.

https://doi.org/10.1002/anie.200901843

5. Gholap, S. S. Eur. J. Med. Chem. 2016, 110, 13-31. https://doi.org/10.1016/i.ejmech.2015.12.017

6. Joshi, S. D.; More, U. A.; Kulkarni,V. H.; Aminabhavi, T. M. Curr. Org. Chem. 2013, 17, 2279-2304. https://doi.org/10.2174/13852728113179990040

7. Schmuck, C.; Rupprecht, D. Synthesis 2007, 3095-3110. https://doi.org/10.1055/s-2007-990783

8. Xiao, D.; Schreier, J. A., Cook, J. H., Seybold, P. G., Ketcha, D. M. Tetrahedron Lett. 1996, 37, 1523-1526 and references cited therein.

https://doi.org/10.1016/0040-4039(96)00098-6

9. Szmuszkovicz, J. J. Am. Chem. Soc. 1957, 79, 2819-2821. 
https://doi.org/10.1021/ja01568a041

10. Iqbal, Z.; Jackson, A. H.; Rao, K. R. N. Tetrahedron Lett. 1988, 29, 2577-2580. https://doi.org/10.1016/S0040-4039(00)86116-X

11. Poupaert, J. H.; Bukuru, J.; Gozzo, A. M. Monatsh. Chem. 1999, 130, 929-932.

12. Harrington, P. E.; Kerr, M. A. Synlett 1996, 1047-1048.

13. Yadav, J. S.; Abraham, S.; Reddy, B. V. S.; Sabitha, G. Synthesis 2001, 2165-2169. https://doi.org/10.1055/s-2001-18068

14. Srivastava, N.; Banik, B. K. J. Org. Chem. 2003, 68, 2109-2114. https://doi.org/10.1021/jo026550s

15. Reddy, A. V.; Ravinder, K.; Goud, T. V.; Krishnaiah, P.; Raju, T. V.; Venkateswarlu, Y. Tetrahedron Lett. 2003, 44, 6257-6260. https://doi.org/10.1016/S0040-4039(03)01555-7

16. Alam, M. M.; Varala, R.; Adapa, S. R. Tetrahedron Lett. 2003, 44, 5115-5119. https://doi.org/10.1016/S0040-4039(03)01089-X

17. Xie, J.; Zhu, X.; Huang, M.; Meng, F.; Wang, M.; Wan, Y. Synth. Commun. 2010, 40, 3259-3267. https://doi.org/10.1080/00397910903398676

18. Manabe, K.; Aoyama, N.; Kobayashi, S. Adv. Synth. Catal. 2001, 343, 174-176. https://doi.org/10.1002/1615-4169(20010226)343:2<174::AID-ADSC174>3.0.CO;2-S

19. Komoto, I.; Kobayashi, S. J. Org. Chem. 2004, 69, 680-688. https://doi.org/10.1021/j00353177

20. Evans, D. A.; Scheidt, K. A.; Fandrick, K. R.; Lam, H. W.; Wu , J. J. Am. Chem. Soc. 2003, 125, 10780-10781. https://doi.org/10.1021/ja036985c

21. Wang, S.-Y.; Ji, S.-J.; Loh, T.-P. Synlett 2003, 2377-2379.

22. Banik, B. K.; Fernandez, M.; Alvarez, C. Tetrahedron Lett. 2005, 46, 2479-2482. https://doi.org/10.1016/j.tetlet.2005.02.044

23. Liang, D.; Li, X.; Zhang, W.; Li, Y.; Zhang, M.; Cheng, P. Tetrahedron Lett. 2016, 57, 1027-1030. https://doi.org/10.1016/i.tetlet.2016.01.078

24. Swetha, A.; Reddy, M. R.; Babu, B. M.; Meshram, H. M. Tetrahedron Lett. 2017, 58, 4427-4431. https://doi.org/10.1016/i.tetlet.2017.09.016

25. Ji, S.-J.; Wang, S.-Y. Synlett 2003, 2074-2076. https://doi.org/10.1055/s-2003-41463

26. Bartoli, G.; Bartolacci, M.; Bosco, M.; Foglia, G.; Giuliani, A.; Marcantoni, E.; Sambri, L.; Torregiani, E. J. Org. Chem. 2003, 68, 4594-4597. https://doi.org/10.1021/jo034303y

27. Huang, Z. H.; Zou, J. P.; Jiang, W. Q. Tetrahedron Lett. 2006, 47, 7965-7968. https://doi.org/10.1016/i.tetlet.2006.08.108

28. Xu, R.; Ding, J. C.; Chen, X. A.; Liu, M. C.; Wu, H. Y. Chin. Chem. Lett. 2009, 20, 676-679. https://doi.org/10.1016/i.cclet.2009.01.028

29. Gu, Y.; Ogawa, C.; Kobayashi, S. Org. Lett. 2007, 9, 175-178. https://doi.org/10.1021/ol062446b

30. Yadav, J. S.; Abraham, S.; Reddy, B. V. S.; Sabitha, G. Tetrahedron Lett. 2001, 42, 8063-8065. https://doi.org/10.1016/S0040-4039(01)01697-5

31. Unaleroglu, C.; Temelli, B.; Demir, A. S. Synthesis 2004, 2574-2578. https://doi.org/10.1055/s-2004-831195 
32. Cavdar, H.; Saracoglu, N. Tetrahedron 2005, 61, 2401-2405. https://doi.org/10.1016/i.tet.2005.01.017

33. Kusurkar, R. S.; Nayak, S. P.; Chavan, N. L. Tetrahedron Lett. 2006, 47, 7323-7326. https://doi.org/10.1016/j.tetlet.2006.08.030

34. Das, B.; Damodar, K.; Chowdhury, N. J. Mol. Catal. A: Chem. 2007, 269, 81-84. https://doi.org/10.1016/j.molcata.2007.01.007

35. Firouzabadi, H.; Iranpoor, N.; Nowrouzi, F. Chem. Commun. 2005, 789-791. https://doi.org/10.1039/B412653J

36. Zhan, Z.-P.; Yu, J.-L.; Yang, W.-Z. Synth. Commun. 2006, 36, 1373-1382. https://doi.org/10.1080/00397910500522074

37. Zhan, Z.-P.; Yang, W.-Z.; Yang, R.-F. Synlett 2005, 2425-2428. https://doi.org/10.1055/s-2005-872688

38. Habib, P. M.; Kavala, V.; Kuo, C.-W.; Riahan, M. J.; Yao, C.-F. Tetrahedron 2010, 66, 7050-7056. https://doi.org/10.1016/i.tet.2010.05.104

39. de Rosa, M.; Soriente, A. Tetrahedron 2010, 66, 2981-2986. https://doi.org/10.1016/i.tet.2010.02.055

40. Shumaila, A. M. A.; Kursurkar, R. S. Synth. Commun. 2010, 40, 2935-2940. https://doi.org/10.1080/00397910903340694

41. Kusurkar, R. S.; Alkobati, N. A. H. Synth. Commun. 2010, 40, 320-327. https://doi.org/10.1080/00397910903458025

42. Hari, G. S.; Nagaraju, M.; Murthy, M. M. Synth. Commun. 2008, 38, 100-105. https://doi.org/10.1080/00397910701650815

43. Zolfigol, M. A.; Veisi, H.; Mohanazadeh, F.; Sedrpoushan, A. J. Heterocycl. Chem. 2011, 48, 977-986. https://doi.org/10.1002/jhet.659

44. Kappe, C.O. Angew. Chem. Int. Ed. 2004, 43, 6250-6284. https://doi.org/10.1002/anie.200400655

45. Kappe, C. O.; Dallinger, D.; Murphree, S. S. Practical Microwave Synthesis for Organic Chemists; VCH: Weinheim, 2008. https://doi.org/10.1002/9783527623907

46. Miles, K. C.; Mays, S .M.; Southerland, B. K., Auvil, T .J.; Ketcha, D. M. Arkivoc 2009, (xiv), 181-190. http://dx.doi.org/10.3998/ark.5550190.0010.e17

47. Ketcha, D. M.; Carpenter, K. P.; Atkinson, S. T.; Rajagopalan, H. R. Synth. Commun. 1990, 20, 1647-1655. https://doi.org/10.1080/00397919008053085

48. Saulnier, M. G.; Gribble, G.W. J. Org. Chem. 1982, 47, 757-761. https://doi.org/10.1021/jo00344a001

49. Gribble, G. W. "Pyrroles and their benzo derivatives: applications" in Comprehensive Heterocyclic Chemistry II, Ed. Bird, C. W. Elsevier: Oxford, 1996; Vol. 2, pp 207-257. 